\title{
Percutaneous coronary intervention in diabetic versus non-diabetic patients with prior coronary artery bypass grafting: a propensity score matching study
}

Xiaolong Ma', Ran Dong ${ }^{1}$, Pengfei Chen ${ }^{1}$, Yichen Zhao ${ }^{1}$, Caiwu Zeng ${ }^{2}$, Meng Xin², Qing Ye ${ }^{1}$ and Jiangang Wang ${ }^{1 *}$

\begin{abstract}
Background: The target of this study was to explore the outcomes of percutaneous coronary intervention (PCI) in diabetic versus non-diabetic patients with prior coronary artery bypass grafting (CABG) surgery.

Methods: Seven hundred and twenty four patients who had previously received CABG and had been treated using $\mathrm{PCl}$ combined with drug-eluting stents (DES) between 2009 and 2017 were selected for a retrospective study and allocated into either a diabetes mellitus (DM) or non-diabetes mellitus (No DM) group. A 1:1 propensity scorematched evaluation was conducted and risk adjusted for analysis. The primary outcomes were cardiac death, myocardial infarction, heart failure and revascularization, with a median follow-up duration of 5.13 years.

Results: After matching, two-, 5- and 8-year event rate of overall major adverse cardiac events (MACEs) were found to be higher in the DM group (No DM vs DM:15.3, 30.9, 38.5\% vs 19.8, 37.8, 52.2\%, respectively), although no significant difference was found in the event rate of overall MACEs (hazard ratio [HR]: 1.35; 95\% confidence interval [CI]: 1.00 to 1.83 for DM vs No DM; $P=0.052$ ), cardiac death (HR: 0.94; $95 \% \mathrm{Cl}: 0.45$ to $1.95 ; P=0.871)$, MI (HR: $1.49 ; 95 \%$ Cl: 0.95 to 2.32; $P=0.080$ ), HF (HR: $1.54 ; 95 \% \mathrm{Cl}: 0.90$ to 2.63 for; $P=0.120$ ) or revascularization (HR: $1.07 ; 95 \%$ Cl: 0.72 to $1.59 ; P=$ 0.747). Subgroup analysis of PCl in only the NCA showed MACEs (adjusted HR: 1.13; $95 \%$ Cl: 0.85 to 1.49 for DM vs No $\mathrm{DM} ; P=0.325$ ), cardiac death (adjusted HR: $0.85 ; 95 \% \mathrm{Cl}: 0.41$ to 1.78 for DM vs No DM; $P=0.781$ ), Ml (adjusted HR: 1.32; 95\% Cl: 0.84 to 2.01 for DM vs No DM; $P=0.069$ ), HF (adjusted HR: 1.41; $95 \%$ Cl: 0.87 to 2.27 for DM vs No DM; $P=$ 0.211 ) or repeated revascularization (adjusted HR: 0.93; $95 \%$ Cl: 0.64 to 1.37 for DM vs No DM; $P=0.836$ ).
\end{abstract}

Conclusions: Compared with non-diabetic patients with prior CABG, subsequent implantation of DES in the native coronary artery of diabetic patients resulted in apparently similar outcomes.

Trial registration: This study was not registered in an open access database.

Keywords: Percutaneous coronary intervention, Diabetic, Non-diabetic, Prior coronary artery bypass grafting, Outcomes

\footnotetext{
* Correspondence: jiangangwang@ccmu.edu.cn

'Department of Cardiac Surgery, Beijing Anzhen Hospital, Capital Medical University, Beijing, China

Full list of author information is available at the end of the article
}

(c) The Author(s). 2020 Open Access This article is licensed under a Creative Commons Attribution 4.0 International License, which permits use, sharing, adaptation, distribution and reproduction in any medium or format, as long as you give appropriate credit to the original author(s) and the source, provide a link to the Creative Commons licence, and indicate if changes were made. The images or other third party material in this article are included in the article's Creative Commons licence, unless indicated otherwise in a credit line to the material. If material is not included in the article's Creative Commons licence and your intended use is not permitted by statutory regulation or exceeds the permitted use, you will need to obtain permission directly from the copyright holder. To view a copy of this licence, visit http://creativecommons.org/licenses/by/4.0/. The Creative Commons Public Domain Dedication waiver (http://creativecommons.org/publicdomain/zero/1.0/) applies to the data made available in this article, unless otherwise stated in a credit line to the data. 


\section{Background}

Diabetic patients have a higher prevalence of coronary artery disease (CAD) than the general population, manifesting as diffuse lesions and severe atherosclerosis in the left main artery and multiple other vessels [1], severe symptoms often developing earlier in life combined with a substantially poorer prognosis than non-diabetic patients $[2,3]$. It has been reported that diabetes is considered a predictor of adverse events such as myocardial infarction (MI), repeat revascularization and cardiac death for patients who have undergone coronary artery bypass grafting (CABG) [4-6].

For patients with prior CABG who require repeat revascularization, percutaneous coronary intervention (PCI) is usually the preferred strategy, rather than redo CABG, because of the low procedural mortality and similar long-term outcome $[7,8]$, combined with placement of a drug-eluting stent (DES) [9]. Despite a number of studies investigating the impact that diabetes has on the clinical outcome of PCI with DES in patients without prior CABG $[10,11]$, little is known about the influence of diabetes on outcomes of PCI with DES in patients who have previously undergone CABG.

In this study, we retrospectively assessed the clinical data of non-diabetic and diabetic patients with prior CABG who had subsequently received PCI with DES, aiming to establish the impact of DM on the long-term outcomes of PCI for restenosis after CABG.

\section{Methods}

\section{Study design}

This study was a retrospective observational study conducted in Beijing Anzhen Hospital, Capital Medical University, Beijing Institute of Heart Lung and Blood Vessel Diseases, Beijing, China. A total of 724 patients with prior CABG were selected from the institution's PCI registry (2009 to 2017) who had undergone PCI with DES in a native coronary artery (NCA), following CABG surgery for coronary atherosclerotic heart disease in the same hospital. Patients were segregated into a diabetes mellitus (DM) or non-diabetes mellitus (No DM) group, according to whether or not they suffered from DM. All data were reviewed by one cardiac surgeon and two cardiologists, the latter contacting patients for follow-up outcomes by telephone, mail or visit. The study was approved by the Institutional Ethics Committee of Beijing Anzhen Hospital.

\section{Definitions used in this study}

Patients' data before PCI was defined as baseline data. DM was defined as either a previous diagnosis of DM treated with diet, oral agents, peptide analogs or insulin, or a new diagnosis after index hospitalization [12] before PCI. A graft with a stenosis of $>70 \%$ of its diameter was defined as stenosis. A graft with stenosis or occlusion was classified as a diseased graft in this study. The classification of ischemic territory was based on the results of coronary angiography (CAG) after re-hospitalization and also referred to the results prior to CABG. NCA related to ischemic territory was defined as relevant NCA. Paclitaxel-eluting and sirolimuseluting stents were defined as first-generation DES. Everolimus-eluting and zotarolimus-eluting stents were defined as second-generation DES. Procedural complications refer to complications post PCI. PCI failure was defined as failure to implant a stent at one lesion site.

The primary end-point was a major adverse cardiac event (MACE), defined as the combined incidence of either cardiac death, myocardial infarction (MI), heart failure (HF) or revascularization, as independently adjudicated by an events committee. Cardiac death was defined as any death due to MI, HF, lethal arrhythmia or sudden death in a previously stable patient [13]. MI was defined as: (1) elevation of myocardial enzymes such as cardiac troponin $\mathrm{T}$ (cTnT) or creatine kinase-muscle/brain (CK-MB) $>2$ fold higher than the upper normal value and (2) changes in ST-segment and T-wave (ST-T) on electrocardiography [14]. HF was defined as hospitalization for progressive heart failure with clinical and radiographic signs. Revascularization was defined as undergoing a subsequent revascularization procedure by PCI or Redo CABG after discharge from the Department of Cardiology, Anzhen Hospital.

\section{Statistical analysis}

All results were analyzed using Stata SE for Windows, version 15.0 (Stata Corporation, College Station, TX, USA) statistical package and IBM SPSS Statistics for Windows, version 22.0. Categorical variables are presented as raw numbers (\%) and continuous variables as means \pm standard deviation. Comparisons of the DM and No DM groups were accomplished using a Fisher's exact test for each variable and Mann-Whitney-Wilcox nonparametric test for continuous variables. To reduce the impact of potential confounding on MACEs on the results of the observational study, 1:1 propensity score matching was conducted to choose patients with comparable baseline data. After evaluation of covariates associated clinically and / or statistically with the treatment group and removal of repeatedly defined or collinear variables, including baseline characteristics, risk factors, medical history, clinical conditions at admission and treatment during hospitalization, 36 variables listed in Fig. 2 were included in the propensity score matching model using greedy nearest neighbor matching without replacement and a caliper of 0.02 . The absolute standardized difference in variables included for the calculation of propensity score were compared before and after propensityscore matching. The absolute standardized difference cutoff point for the variables included in the calculation was fixed at $10.0 \%$. After matching, Cox proportional hazards regression analysis was also conducted to assess the 
association between variables and follow-up outcomes. Univariable Cox proportional hazards regression models were initially conducted, followed by multivariable Cox proportional hazards regression models. The candidate variables were potential confounding variables that were either mostly included in the propensity score matching model or reported more than once with an effect on cardiac death or MACEs. After forward stepwise selection with inclusion criteria both set at $P=0.2$, the variables were eventually included in multivariable Cox proportional hazards regression models of cardiac death and MACEs, respectively. Outcomes were compared using a log-rank test and presented as Kaplan-Meier curves. For all analyses reported, $P$ values were 2 -sided. Statistical differences were considered significant for values of $P<0.05$.

\section{Results}

\section{Baseline characteristics}

In this study, 724 patients were included of which 351 patients (48.5\%) exhibited DM. In the DM group, 43.3\% of patients presented between 1 and 5 years after CABG and $33.0 \%$ between 5 and 10 years, as shown in Fig. 1. The absolute standardized difference values before and after matching are shown in Fig. 2. Following the matching, absolute standardized differences $<10.0 \%$ for those variables included indicated a relatively small imbalance.

The baseline characteristics are shown in Table 1. After matching, there were 256 patients in each group, and the statistical difference in age (years), BMI, duration of period after CABG, numbers of patients that were $>65$ years old, had chronic lung disease, prior cerebrovascular accidents (CVA) or prior PCI were not as significant as they had been before matching the two groups. The characteristics of the CAGs of the diseased grafts and relevant NCAs are

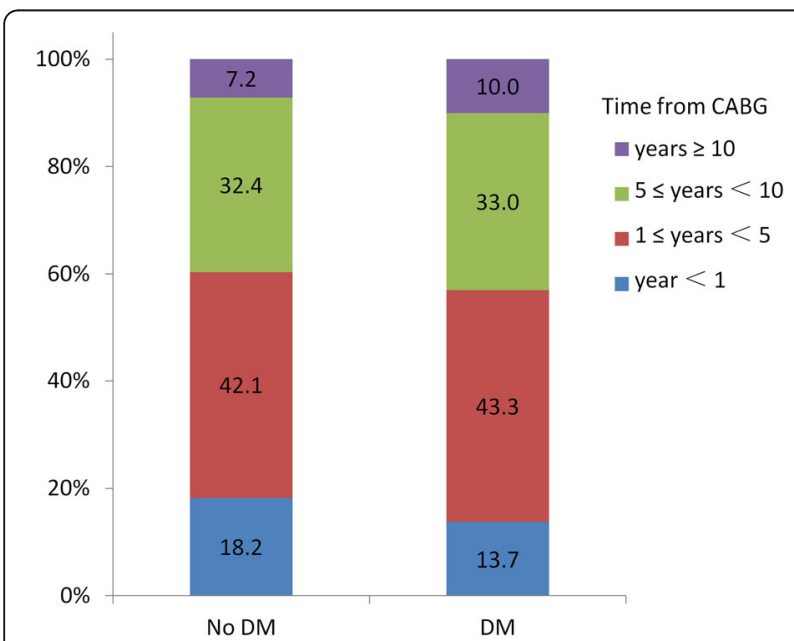

Fig. 1 Distribution of patients accepting subsequent $P C I$ with DESS by time period after coronary artery bypass graft surgery, $P=0.261$. $\mathrm{PCl}=$ percutaneous coronary intervention, $\mathrm{DESs}=$ drug-eluting stents also shown in Table 1, with no significant difference between the two groups before matching. The relevant indicators of blood sugar of patients such as glucose concentratrion and glycosylated hemoglobin (HbA1c) are also shown in Table 1.

\section{PCl-related baseline characteristics}

Table 2 displays the procedural baseline characteristics, which are also included in the propensity score matching. After matching, PCI was mostly performed in NCA only (No DM: $87.1 \%$ vs DM: $87.9 \%$ ) with first-generation DES used widely (No DM: 62.1\% vs DM: $64.8 \%$ ) in each group. A small proportion of patients also underwent percutaneous coronary angioplasty (PTCA) in addition to stent implantation (No DM: $7.4 \%$ vs DM: $9.0 \%$ ) and PCI failure in stent implantation in one lesion site (No DM: $4.3 \%$ vs DM: $5.1 \%$ ). EPD, rotational atherectomy, aspiration of thrombus or intravascular ultrasound (IVUS) were not greatly used in either group.

\section{Procedural complications}

Table 3 describes recorded procedural complications. After matching, the DM group exhibited slightly high in-hospital mortality, with a higher incidence of angina after $24 \mathrm{~h}(5.1 \%)$, periprocedural MI (1.2\%), stroke (0.8\%) and bleeding (1.6\%), but there was no significant difference between the two groups.

\section{Follow-up outcomes}

Complete follow-up data were obtained in the overall study population, as displayed in Table 4 and Fig. 3. The median follow-up duration was 5.13 years. After matching, KaplanMeier curves (Fig. 4a) indicated that the cumulative overall rate of MACEs was higher in the DM group at 2 years (No DM: $15.3 \%$ vs DM: $19.8 \%$ ), 5 years (No DM: $30.9 \%$ vs DM: $37.8 \%$ ) and at 8 years (No DM: $38.5 \%$ vs DM: $52.2 \%$ ) (hazard ratio [HR]: 1.35 ; $95 \%$ confidence interval $[\mathrm{CI}]: 1.00$ to 1.83 for DM vs No DM; $P=0.052$ ), with an apparent increase in the difference between the two groups over time. The curves indicated that two-, 5- and 8-year incidence of cardiac death in each group (Fig. 4b) were similar (No DM: 1.6, 5.8, 10.5\% vs DM 1.2, 5.8, 9.3\%; HR: 0.94; 95\% CI: 0.45 to 1.95 for DM vs No DM; $P=0.871$ ). There was also no statistical difference in incidence of MI (HR: 1.49; 95\% CI: 0.95 to 2.32 for DM vs No DM; $P=0.080$ ), HF (HR: 1.54 ; 95\% CI: 0.90 to 2.63 for DM vs No DM; $P=0.120$ ) or repeated revascularization (HR: 1.07 ; $95 \%$ CI: 0.72 to 1.59 for $\mathrm{DM}$ vs No DM; $P=0.747$ ) between the two groups, although there was a trend that the incidence of MI or HF in the DM group increased over time.

Multivariable Cox proportional hazards regression (Fig. 4) demonstrated that patients with hypertension (adjusted HR: 1.85; 95\% CI: 1.32 to 2.59; $\mathrm{P}<0.001$ ), aspiration of thrombus during PCI (adjusted HR: 3.37; 95\% 


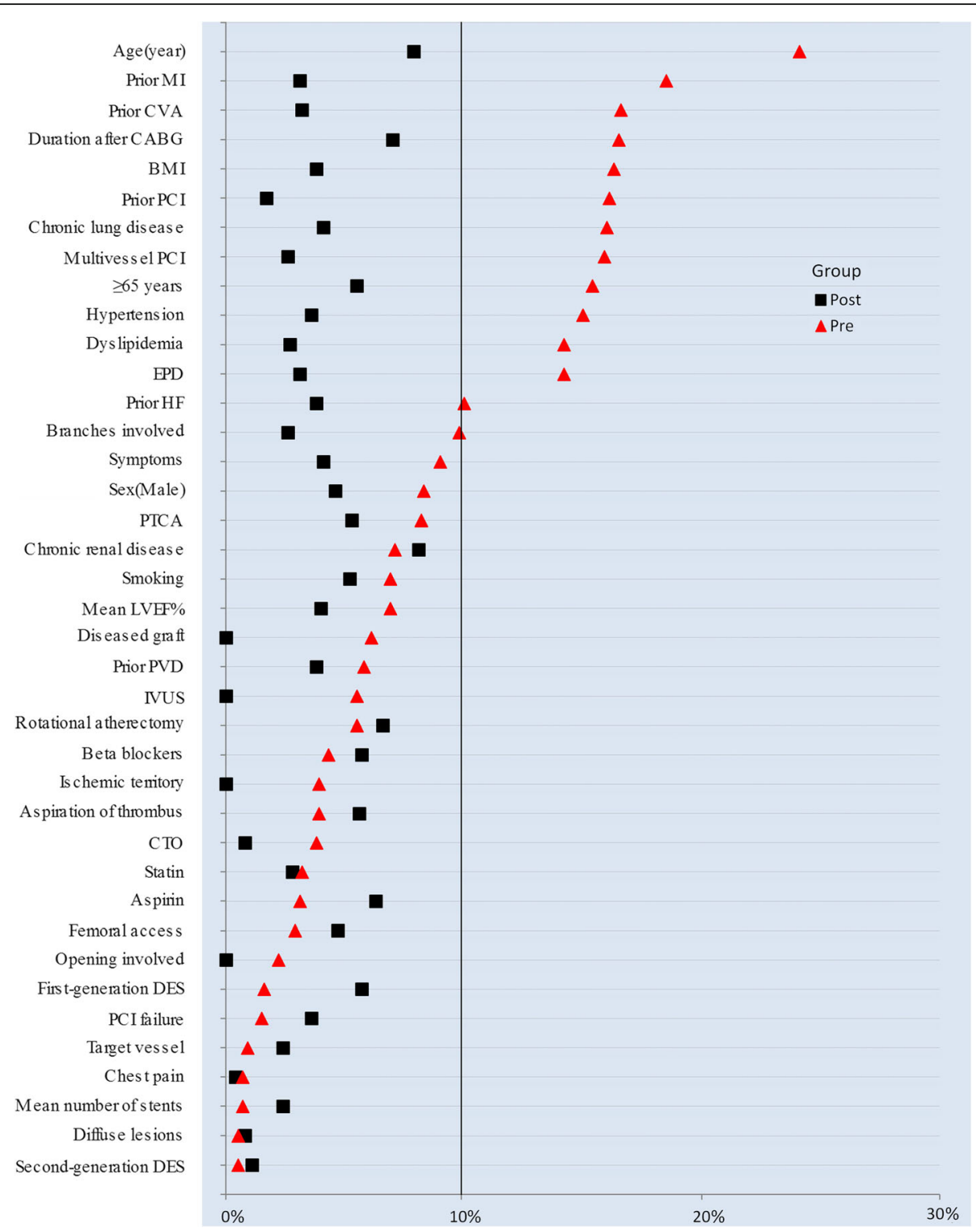

Fig. 2 Dotplot of absolute standardized differences before and after matching

CI: 1.05 to $10.84 ; P=0.041$ ), prior $\mathrm{HF}$ (adjusted HR: 4.30; $95 \%$ CI: 1.82 to $10.14 ; P=0.001$ ) or $\mathrm{LVEF}<50 \%$ (adjusted HR: 1.52 ; $95 \% \mathrm{CI}: 1.07$ to $2.17 ; P=0.020$ ) were more likely to suffer a MACE. Additionally, use of a statin provided protection from MACEs (adjusted HR: 0.57; 95\% CI: 0.41 to $0.78 ; P=0.001)$.

\section{Subgroup analysis of $\mathrm{PCl}$ in different target vessels}

To reduce the influence of PCI in different target vessels, we performed two additional subgroup analyses of patients that had undergone PCI only in the NCA or PCI in both the NCA and graft. The baseline characteristics of the two subgroups are detailed within Additional file 1: Tables S1, S2 and S3. The follow-up outcomes of the two subgroups are provided separately in Tables S4, S5 and Figures S1, S2.

The follow-up outcomes of patients that underwent PCI in only the NCA were consistent with all clinical endpoints experienced by the whole population. Compared with the non-diabetic patients who had previously undergone CABG, subsequent PCI in only the NCA in diabetic patients appeared to result in similar outcomes (Table S4, Figure S1), including MACEs (adjusted HR: 1.13; $95 \%$ CI: 0.85 to 1.49 for DM vs No DM; $P=0.325$ ), cardiac death (adjusted HR: 0.85; 95\% CI: 0.41 to 1.78 for DM vs No DM; $P=0.781$ ), MI (adjusted HR: 1.32; 95\% CI: 0.84 to 2.01 for DM vs No DM; $P=0.069)$, HF (adjusted HR: 1.41 ; $95 \%$ CI: 0.87 to 2.27 for DM vs No DM; $P=0.211$ ) or repeated revascularization (adjusted 
Table 1 Comparison of baseline characteristics of non-diabetic vs diabetic patients with prior CABG $(n=724)$

\begin{tabular}{|c|c|c|c|c|c|c|}
\hline \multirow[t]{3}{*}{ Variable } & \multicolumn{3}{|l|}{ Unmatched } & \multicolumn{3}{|l|}{ Matched } \\
\hline & \multirow{2}{*}{$\begin{array}{l}\text { No DM } \\
n=373\end{array}$} & \multirow{2}{*}{$\begin{array}{l}\mathrm{DM} \\
n=351\end{array}$} & \multirow[t]{2}{*}{$P$ value } & \multirow{2}{*}{$\begin{array}{l}\text { No DM } \\
n=256\end{array}$} & \multirow{2}{*}{$\begin{array}{l}\mathrm{DM} \\
n=256\end{array}$} & \multirow[t]{2}{*}{$P$ value } \\
\hline & & & & & & \\
\hline \multicolumn{7}{|l|}{ Demographics } \\
\hline Age (year) & $61.52 \pm 9.23$ & $63.64 \pm 8.15$ & 0.001 & $62.25 \pm 8.71$ & $62.86 \pm 8.13$ & 0.409 \\
\hline$\geq 65$ years & 146(39.1\%) & $165(47.0 \%)$ & 0.036 & $101(39.5 \%)$ & $108(42.2 \%)$ & 0.590 \\
\hline Sex (Male) & $290(77.7 \%)$ & $261(74.4 \%)$ & 0.296 & 192(75.0\%) & 197(77.0\%) & 0.679 \\
\hline \multicolumn{7}{|l|}{ Comorbidities } \\
\hline Hypertension & $263(70.5 \%)$ & $268(76.4 \%)$ & 0.078 & 188(73.4\%) & 192(75.0\%) & 0.762 \\
\hline Dyslipidemia & $167(44.8 \%)$ & 182(51.9\%) & 0.063 & $131(51.2 \%)$ & $127(49.6 \%))$ & 0.791 \\
\hline Chronic renal disease & $12(3.2 \%)$ & $16(4.6 \%)$ & 0.441 & $10(3.9 \%)$ & $6(2.3 \%)$ & 0.447 \\
\hline Chronic lung disease & $20(5.4 \%)$ & $8(2.3 \%)$ & 0.035 & 10(3.9\%) & $8(3.1 \%)$ & 0.811 \\
\hline Prior PVD & $43(11.5 \%)$ & $34(9.7 \%)$ & 0.470 & $24(9.4 \%)$ & $27(10.5 \%)$ & 0.768 \\
\hline Prior CVA & 49(13.1\%) & $66(18.8 \%)$ & 0.042 & $40(15.6 \%)$ & $37(14.5 \%)$ & 0.805 \\
\hline Prior Ml & $52(13.9 \%)$ & $75(21.4 \%)$ & 0.011 & $43(16.8 \%)$ & $40(15.6 \%)$ & 0.811 \\
\hline Prior HF & $6(1.6 \%)$ & $2(0.6 \%)$ & 0.288 & $3(1.2 \%)$ & $2(0.8 \%)$ & 1.000 \\
\hline Prior PCl & 14(3.8\%) & $26(7.4 \%)$ & 0.035 & $13(5.1 \%)$ & $14(5.5 \%)$ & 1.000 \\
\hline Smoking & 238(63.8\%) & $212(60.4 \%)$ & 0.358 & $162(63.3 \%)$ & $167(65.2 \%)$ & 0.712 \\
\hline BMI & $25.70 \pm 3.00$ & $26.19 \pm 3.18$ & 0.037 & $25.97 \pm 2.98$ & $25.96 \pm 3.23$ & 0.965 \\
\hline $\mathrm{HbA} 1 \mathrm{c}(\%)$ & $6.02 \pm 1.08$ & $7.50 \pm 1.68$ & $<0.001$ & $5.97 \pm 1.01$ & $7.48 \pm 1.37$ & $<0.001$ \\
\hline Blood sugar (mmol/L) & $5.59 \pm 1.46$ & $7.89 \pm 3.04$ & $<0.001$ & $5.43 \pm 0.83$ & $7.86 \pm 2.77$ & $<0.001$ \\
\hline Symptoms & & & 0.245 & & & 0.811 \\
\hline Chest pain & $356(95.4 \%)$ & $341(97.2 \%)$ & 0.456 & 248(96.9\%) & 246(96.1\%) & 0.966 \\
\hline SA & 142(39.9\%) & $126(37.0 \%)$ & & $98(39.5 \%)$ & $96(39.0 \%)$ & \\
\hline UA & 133(37.4\%) & 124(36.4\%) & & $90(36.3 \%)$ & $92(37.4 \%)$ & \\
\hline AMl & $81(22.8 \%)$ & $91(26.7 \%)$ & & $60(24.2 \%)$ & $58(23.6 \%)$ & \\
\hline Others & $17(4.6 \%)$ & $10(2.8 \%)$ & & $8(3.1 \%)$ & 10(3.9\%) & \\
\hline Mean LVEF\% & $59.13 \pm 9.16$ & $58.46 \pm 9.29$ & 0.364 & $59.06 \pm 8.96$ & $58.65 \pm 9.03$ & 0.631 \\
\hline Duration after CABG & $4.48 \pm 3.43$ & $5.03 \pm 3.66$ & 0.034 & $4.68 \pm 3.43$ & $4.71 \pm 3.52$ & 0.917 \\
\hline \multicolumn{7}{|l|}{ CAG characteristics } \\
\hline Diseased graft & $303(81.2 \%)$ & 278(79.2\%) & 0.514 & $207(80.9 \%)$ & 207(80.9\%) & 1.000 \\
\hline \multicolumn{7}{|l|}{ Relevant NCAs } \\
\hline СТО & 194(52.0\%) & $176(50.1 \%)$ & 0.655 & $128(50.0 \%)$ & $127(49.6 \%)$ & 1.000 \\
\hline Diffuse lesions & $56(15.0 \%)$ & $53(15.1 \%)$ & 1.000 & $38(14.8 \%)$ & $37(14.5 \%)$ & 1.000 \\
\hline Branches involved & 104(27.9\%) & $114(32.5 \%)$ & 0.195 & $70(27.3 \%)$ & $73(28.5 \%)$ & 0.844 \\
\hline Opening involved & 117(31.4\%) & 106(30.2\%) & 0.748 & 81(31.6\%) & $81(31.6 \%)$ & 1.000 \\
\hline Ischemic territory & & & 0.885 & & & 0.649 \\
\hline One territory & 142(38.1\%) & 137(39.0\%) & & 102(39.8\%) & 99(38.7\%) & \\
\hline Two territories & 172(46.1\%) & 163(46.4\%) & & $125(48.8 \%)$ & $121(47.3 \%)$ & \\
\hline Three territories & 59(15.8\%) & $51(14.5 \%)$ & & 29(11.3\%) & $36(14.1 \%)$ & \\
\hline SYNTAX Score I & $42.0 \pm 13.0$ & $43.4 \pm 12.7$ & 0.145 & $42.1 \pm 13.1$ & $42.8 \pm 12.9$ & 0.575 \\
\hline
\end{tabular}

AMI Acute myocardial infarction, BMI Body mass index, CABG Coronary artery bypass graft, CTO Chronic total occlusion, CVA Cerebrovascular accident, DM Diabetes mellitus, HbA1c Glycosylated hemoglobin, HF Heart failure, LVEF Left ventricular ejection fraction, NCA Native coronary artery, $P C I$ Percutaneous coronary intervention, PVD Peripheral vascular disease, SA Stable angina, SVG Saphenous vein graft, UA Unstable angina 
Table 2 Procedural baseline characteristics of patients with prior CABG $(n=724)$

\begin{tabular}{|c|c|c|c|c|c|c|}
\hline \multirow[t]{3}{*}{ Variable } & \multicolumn{3}{|l|}{ Unmatched } & \multicolumn{3}{|l|}{ Matched } \\
\hline & \multirow{2}{*}{$\begin{array}{l}\text { No DM } \\
n=373\end{array}$} & \multirow{2}{*}{$\begin{array}{l}\text { DM } \\
n=351\end{array}$} & \multirow{2}{*}{$\begin{array}{l}P \\
\text { value }\end{array}$} & \multirow{2}{*}{$\begin{array}{l}\text { No DM } \\
n=256\end{array}$} & \multirow{2}{*}{$\begin{array}{l}\mathrm{DM} \\
n=256\end{array}$} & \multirow{2}{*}{$\begin{array}{l}P \\
\text { value }\end{array}$} \\
\hline & & & & & & \\
\hline Femoral access & $201(53.9 \%)$ & $185(52.7 \%)$ & 0.766 & 138(53.9\%) & $132(51.6 \%)$ & 0.658 \\
\hline Target vessel & & & 1.000 & & & 0.894 \\
\hline NCA only & $327(87.7 \%)$ & $308(87.7 \%)$ & & $223(87.1 \%)$ & $225(87.9 \%)$ & \\
\hline NCA and Graft & $46(12.3 \%)$ & $43(12.3 \%)$ & & $35(12.9 \%)$ & $32(12.1 \%)$ & \\
\hline Multi-vessel PCl & $122(32.7 \%)$ & $90(25.6 \%)$ & 0.041 & $73(28.5 \%)$ & $70(27.3 \%)$ & 0.844 \\
\hline \multicolumn{7}{|l|}{ Stent } \\
\hline Mean number of stents & $1.88 \pm 1.13$ & $1.87 \pm 1.09$ & 0.874 & $1.83 \pm 1.11$ & $1.89 \pm 1.12$ & 0.558 \\
\hline First-generation DES & 236(63.3\%) & $220(62.7 \%)$ & 0.878 & $159(62.1 \%)$ & $166(64.8 \%)$ & 0.582 \\
\hline Second-generation DES & $159(42.6 \%)$ & 147(41.9\%) & 0.880 & $107(41.8 \%)$ & $106(41.4 \%)$ & 1.000 \\
\hline PTCA & $40(10.7 \%)$ & $30(8.5 \%)$ & 0.379 & 19(7.4\%) & $23(9.0 \%)$ & 0.629 \\
\hline $\mathrm{PCl}$ failure & $18(4.8 \%)$ & $19(5.4 \%)$ & 0.739 & $11(4.3 \%)$ & $12(5.1 \%)$ & 0.835 \\
\hline EPD & $12(3.2 \%)$ & $5(1.4 \%)$ & 0.142 & $5(2.0 \%)$ & $4(1.6 \%)$ & 1.000 \\
\hline Rotational atherectomy & $4(1.1 \%)$ & $6(1.7 \%)$ & 0.535 & $4(1.6 \%)$ & $4(1.6 \%)$ & 1.000 \\
\hline Aspiration of thrombus & $2(0.5 \%)$ & $3(0.9 \%)$ & 0.678 & $1(0.4 \%)$ & $1(0.4 \%)$ & 1.000 \\
\hline IVUS & $4(1.1 \%)$ & $6(1.7 \%)$ & 0.535 & $3(1.2 \%)$ & $1(0.4 \%)$ & 0.624 \\
\hline \multicolumn{7}{|l|}{ Medication } \\
\hline Aspirin & 368(98.7\%) & $345(98.3 \%))$ & 0.767 & 253(98.8\%) & 252(98.4\%) & 0.725 \\
\hline Statin & $325(87.1 \%)$ & $300(85.5 \%)$ & 0.519 & $200(78.1 \%)$ & 203(79.3\%) & 0.829 \\
\hline Beta blockers & 289(77.5\%) & 277(78.9\%) & 0.654 & $220(85.9 \%)$ & $221(86.3 \%)$ & 1.000 \\
\hline
\end{tabular}

CABG Coronary artery bypass graft, DES Drug-eluting stent, DM Diabetes mellitus, EPD Embolic protection devices, IVUS Intravascular ultrasound, NCA Native coronary artery, $P C I$ Percutaneous coronary intervention, PTCA Percutaneous coronary angioplasty

HR: 0.93 ; $95 \%$ CI: 0.64 to 1.37 for DM vs No DM; $P=$ $0.836)$.

When it came to the follow-up outcomes of patients with PCI in both the NCA and graft (Table S5, Figure S2), diabetic patients with subsequent PCI in both the NCA and graft were more likely to experience MACEs (adjusted HR: 4.00; 95\% CI: 1.67 to 9.58 for DM vs No
DM; $P=0.003$ ), cardiac death (adjusted HR: 16.04; $95 \%$ CI: 1.58 to 162.50 for DM vs No DM; $P=0.066$ ), MI (adjusted HR: 3.78; 95\% CI: 1.29 to 11.06 for DM vs No DM; $P=0.010$ ), HF (adjusted HR: 7.61; 95\% CI: 1.06 to 54.57 for DM vs No DM; $P=0.091$ ) or repeated revascularization (adjusted HR: 3.36; 95\% CI: 1.05 to 10.68 for DM vs No DM; $P=0.276)$.

Table 3 Procedure-related complications of patients with prior CABG $(n=724)$

\begin{tabular}{|c|c|c|c|c|c|c|}
\hline \multirow[t]{3}{*}{ Outcomes } & \multicolumn{3}{|c|}{ Unmatched } & \multicolumn{3}{|l|}{ Matched } \\
\hline & \multirow{2}{*}{$\begin{array}{l}\text { No DM } \\
n=373\end{array}$} & \multirow{2}{*}{$\begin{array}{l}\text { DM } \\
n=351\end{array}$} & \multirow{2}{*}{$\begin{array}{l}P \\
\text { value }\end{array}$} & \multirow{2}{*}{$\begin{array}{l}\text { No DM } \\
n=256\end{array}$} & \multirow{2}{*}{$\begin{array}{l}\text { DM } \\
n=256\end{array}$} & \multirow{2}{*}{$\begin{array}{l}P \\
\text { value }\end{array}$} \\
\hline & & & & & & \\
\hline In-hospital mortality & $0(0.0 \%)$ & $2(0.6 \%)$ & 0.235 & $0(0.0 \%)$ & $1(0.4 \%)$ & 1.000 \\
\hline \multicolumn{7}{|c|}{ Procedural complications } \\
\hline Dysrhythmia & $1(0.3 \%)$ & $2(0.6 \%)$ & 0.613 & $1(0.4 \%)$ & $1(0.4 \%)$ & 1.000 \\
\hline Angina in $24 \mathrm{~h}$ & $13(3.5 \%)$ & $22(6.3 \%)$ & 0.086 & $6(2.3 \%)$ & $13(5.1 \%)$ & 0.159 \\
\hline Periprocedural MI & $4(1.1 \%)$ & $5(1.4 \%)$ & 0.746 & $2(0.8 \%)$ & $3(1.2 \%)$ & 1.000 \\
\hline AHF & $1(0.3 \%)$ & $3(0.9 \%)$ & 0.359 & $1(0.4 \%)$ & $1(0.4 \%)$ & 1.000 \\
\hline Stroke & $1(0.3 \%)$ & $2(0.6 \%)$ & 0.613 & $0(0.0 \%)$ & $2(0.8 \%)$ & 0.499 \\
\hline Dissection & $1(0.3 \%)$ & $2(0.6 \%)$ & 0.613 & $1(0.4 \%)$ & $1(0.4 \%)$ & 1.000 \\
\hline Acute closure & $0(0.0 \%)$ & $2(0.6 \%)$ & 0.235 & $0(0.0 \%)$ & $1(0.4 \%)$ & 1.000 \\
\hline Bleeding & $2(0.5 \%)$ & $5(1.4 \%)$ & 0.273 & $2(0.8 \%)$ & $4(1.6 \%)$ & 0.686 \\
\hline
\end{tabular}


Table 4 Follow-up outcomes of patients with prior CABG $(n=724)$

\begin{tabular}{|c|c|c|c|c|c|c|}
\hline \multirow[t]{3}{*}{ Outcomes } & \multicolumn{3}{|l|}{ Unmatched } & \multicolumn{3}{|l|}{ Matched } \\
\hline & No DM & $\mathrm{DM}$ & \multirow{2}{*}{$\begin{array}{l}P \\
\text { value }\end{array}$} & No DM & $\mathrm{DM}$ & \multirow{2}{*}{$\begin{array}{l}P \\
\text { valu }\end{array}$} \\
\hline & $n=373$ & $n=351$ & & $n=256$ & $n=256$ & \\
\hline MACEs & 114(30.6\%) & $128(36.5 \%)$ & 0.055 & $75(29.3 \%)$ & $96(37.5 \%)$ & 0.051 \\
\hline Cardiac death & $18(4.8 \%)$ & $18(5.1 \%)$ & 0.695 & 15(5.9\%) & $14(5.5 \%)$ & 0.871 \\
\hline Ml & 43(11.5\%) & $62(17.7 \%)$ & 0.010 & $33(12.9 \%)$ & $47(18.4 \%)$ & 0.078 \\
\hline $\mathrm{HF}$ & $39(10.5 \%)$ & 48(13.7\%) & 0.095 & $22(8.6 \%)$ & $33(12.9 \%)$ & 0.117 \\
\hline Revascularization & $66(17.7 \%)$ & $66(18.8 \%)$ & 0.593 & 47(18.4\%) & $50(19.5 \%)$ & 0.747 \\
\hline
\end{tabular}

CABG Coronary artery bypass graft, DM Diabetes mellitus, HF Acute heart failure, MACEs Major adverse cardiac events, MI Myocardial infarction

\section{Subgroup analysis of $\mathrm{PCl}$ with different generation DESs}

To reduce the influence of PCI with different generation DESs, we performed two additional subgroup analyses of patients that had undergone PCI with first-generation DESs or second-generation DESs. The baseline characteristics of the two subgroups are detailed within Additional file 1: Tables S6, S7 and S8. The follow-up outcomes of the two subgroups are provided separately in Tables S9, S10 and Figures S3, S4.

The follow-up outcomes of patients that underwent PCI with first-generation DESs were consistent with all clinical endpoints experienced by the whole population. Compared with the non-diabetic patients who had previously undergone CABG, subsequent PCI with firstgeneration DESs in diabetic patients appeared to result in similar outcomes (Table S9, Figure S3).

When it came to the follow-up outcomes of patients with PCI with second-generation DESs (Table S10, Figure S4), diabetic patients with subsequent PCI with second-generation DESs were more likely to experience MACEs (adjusted HR: 1.76; 95\% CI: 1.00 to 3.08 for DM vs No DM; $P=0.016$ ), MI (adjusted HR: $1.90 ; 95 \% \mathrm{CI}$ : 0.75 to 4.81 for DM vs No DM; $P=0.038$ ) and HF (adjusted HR: 1.87 ; $95 \%$ CI: 0.72 to 4.89 for DM vs No DM; $P=0.018$ ). The outcomes of cardiac death (adjusted HR: 1.10; $95 \%$ CI: 0.01 to 1.47 for DM vs No DM; $P=0.852$ ) and repeated revascularization (adjusted HR: 1.67; 95\% CI: 0.79 to 3.53 for DM vs No DM; $P=0.305$ ) are similar between two groups.

\section{Follow-up outcomes of patients with incomplete revascularization vs complete revascularization}

We reviewed the coronary angiography files, 37 patients (5.1\%) received incomplete revascularization (IR) by PCI and 687 patients $(94.9 \%)$ received complete revascularization (CR) by PCI after CABG. Follow-up outcomes of patients with IR vs CR are shown in Table S11. Compared with patients with $\mathrm{CR}$, patients with IR are more likely to have MACEs ( $40.5 \%$ vs $33.0 \%)$, cardiac death ( $8.1 \%$ vs $4.8 \%)$ and $\mathrm{MI}(21.6 \%$ vs $14.1 \%)$, though there are no significant differences between two groups.

\section{Discussion}

We performed a retrospective observational study to explore the outcomes of PCI with DES in diabetic vs nondiabetic patients who had previously undergone CABG in our single-center registry. We found that, compared to non-diabetic patients with prior CABG, subsequent PCI within the NCA with DES in diabetic patients appeared to result in a similar overall incidence of MACEs, cardiac death, MI, HF or repeated revascularization, extending our current understanding of the safety and efficacy of DES even in high-risk patients with prior CABG. This suggests that a DES may be considered the default option in these patient populations. In this study we also found that hypertension, prior $\mathrm{HF}, \mathrm{LVEF}<50 \%$ and aspiration of thrombus are predictive of overall MACEs and patients taking statins are less likely to experience MACEs. Our results were based on matching propensity scores, which suggests that our findings are not due to negative confounding.

Diabetic patients with CAD are reported to have dysfunctional endothelial cells, increased atherosclerotic burden and fragile lipid-rich plaques $[15,16]$, microcirculation disorder involving smaller vessels, and prothrombotic and proinflammatory states [17, 18], which are related to progression of NCA disease. It is confirmed that $\mathrm{CAD}$ in diabetic patients appears as diffuse atherosclerosis with chronic total occlusion (CTO), opening and bifurcation lesions or multivessel disease and left main disease [19], leading to fewer amenable options for re-intervention and suboptimal stent expansion [8]. In this study, although the characteristics of the lesions in the NCAs relevant to ischemic territory are similar in both diabetic and non-diabetic patients, those in each group represent a high proportion of the CTO lesions, openings involving lesions, branches involving lesions or diffuse lesions. We consider that this is due to the combined CAD risk factors, such as hypertension, dyslipidemia, diabetes mellitus, fat, smoking and gender. In order to remove the influence of confounding CAD risk factors and to compare between the DM group and No DM group more precisely, we used the propensity score matching method, described in detail in the statistical analysis section. 


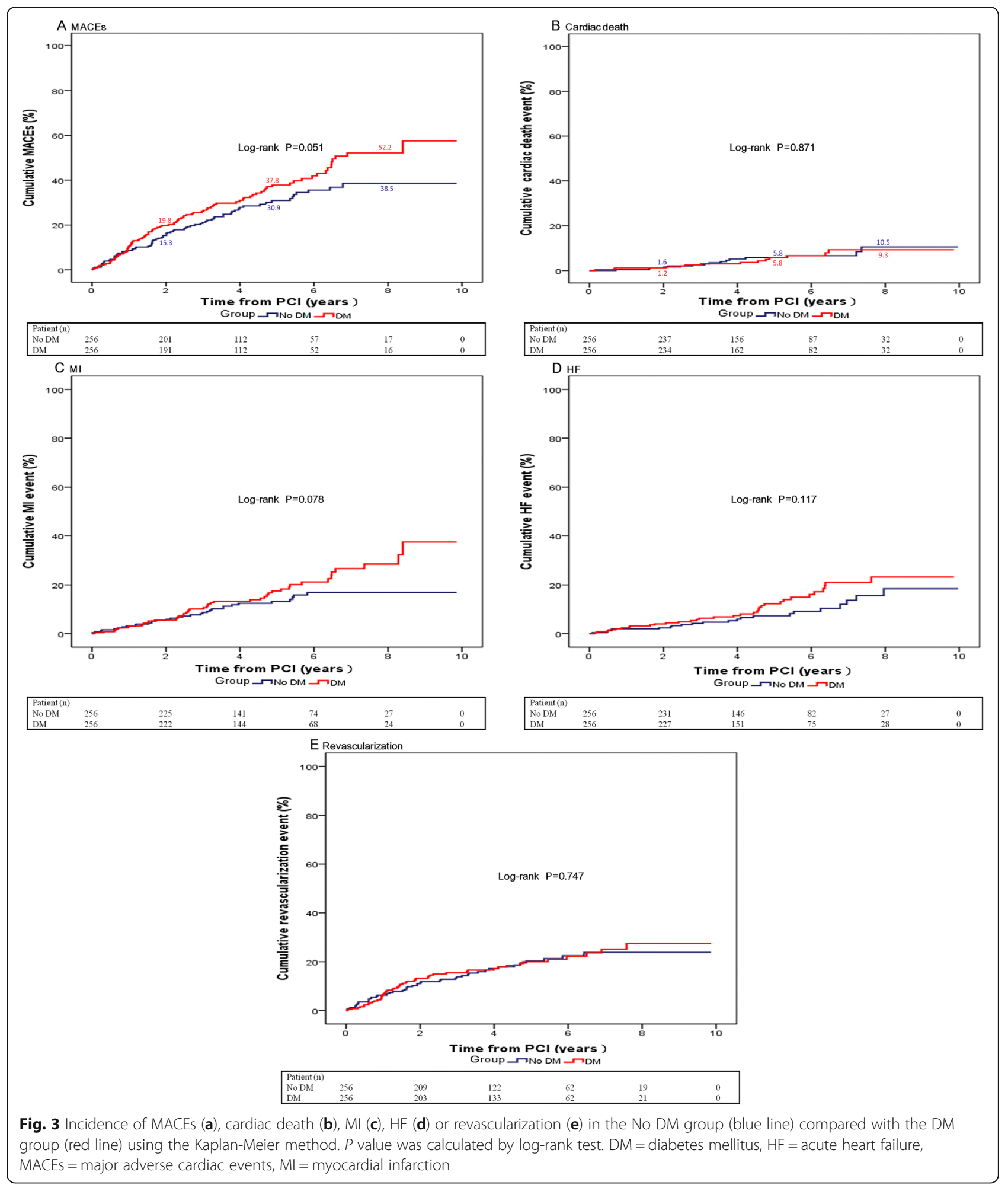

Atherosclerosis is also reported to play an important role in later graft failure (graft age $>6$ months) [8]. Graft atherosclerosis in diabetic patients has a larger necrotic core with unstable plaques [20], which is friable and more prone to distal coronary embolization [2]. Compared with non-diabetic patients with prior CABG, diabetic patients have a higher rate of graft stenosis and recurrent myocardial ischemic events [21, 22], due to the progression of NCA disease or graft failure [2]. In this study the majority of patients in each group had diseased grafts (81.2 and 


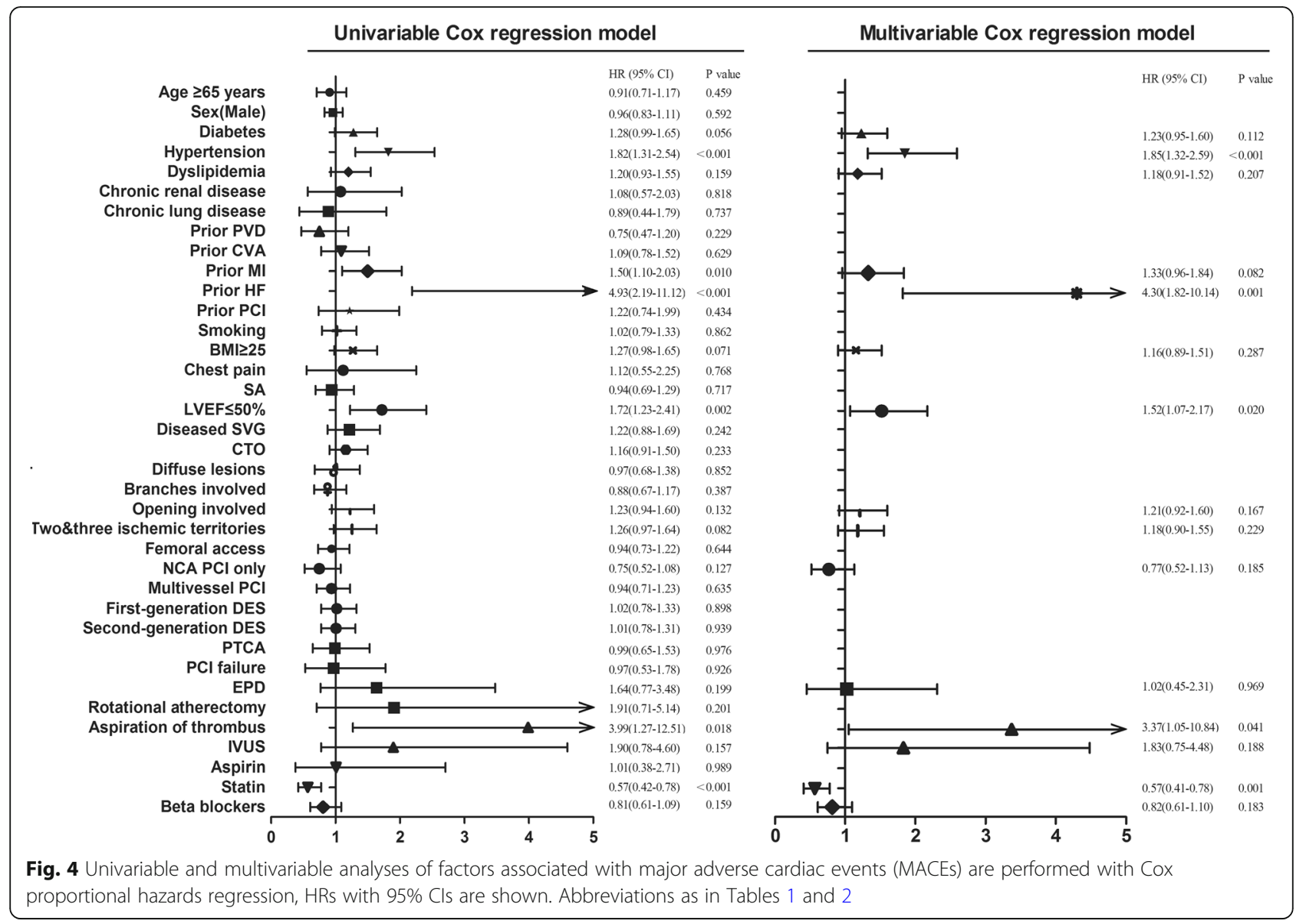

$79.2 \%$ in the No DM and DM groups, respectively), and a small proportion of the remaining patients in each group had myocardial ischema caused by isolated NCA lesions as a consequence of the progression of NCA disease (18.8 and $20.8 \%$ in the No DM and DM groups, respectively).

DES are superior to bare metal stents (BMS), in terms of their strut thickness and polymer coating composition, reducing repeat revascularization and in-stent thrombosis in addition to MI in non-diabetic patients [19]. Published literature indicates that PCI with DES in diabetic patients compared with non-diabetic patients results in significantly higher mortality, reinfarction, and repeat revascularization for in-stent restenosis [23-25]. The pathological mechanism of in-stent restenosis in diabetic patients is associated with excessive endothelial hyperplasia, vascular remodeling or increased platelet aggregation [12]. However, in this study we found different results, especially when performing additional subgroup analysis of patients with PCI in only the NCA, in that PCI in the NCA with DES in diabetic patients compared with non-diabetic patients did not result in a high incidence of cardiac death, HF or repeat revascularization, and the incidence of MI between the two groups was not significantly different. However, one key observation should be clearly noted, that patients in that study included those without prior CABG. Conversely, all patients included in this study underwent prior CABG in our cardiac center, which could be considered a pretreatment for diabetic patients and functions as protection. In addition, all were high risk patients, especially in the DM group. They were older with a greater number of comorbidities and had severe NCA or graft lesions.

The studies of Ahmed [26] and Ashfaq [27] reported the influence of DM on outcomes in saphenous vein graft (SVG) stenting, with similar conclusions, that PCI with DES in diabetics resulted in long-term overall rates of MACEs, death, MI and repeat revascularization that were worse than in non-diabetics, quite different from Pendyala's conclusion that diabetic patients undergoing SVG PCI had similar long-term outcomes [12]. In the present study, after analysis of the whole study population (after matching, $n=512)$, we found that the overall incidence of MACEs (DM: $37.5 \%$ vs No DM: $29.3 \%$ ), principally driven by MI (DM: $18.4 \%$ vs No DM: $12.9 \%)$, were not statistically different between the two groups despite an increasing trend over time. Considering the conflicting data of all the patients that received PCI in the NCA and that a minority of patients were treated with PCI in both graft and NCA (No DM: $12.9 \%$ vs DM: 12.1), we performed further 
subgroup analysis of patients with PCI in only the NCA $(n=635)$. We found that, compared to non-diabetic patients with prior CABG, subsequent DES in only the NCA of diabetic patients appeared to result in similar outcomes, such as rates of MACEs, cardiac death, MI, HF and repeat revascularization. Additional subgroup analysis of patients with PCI in both the NCA and graft, despite the small sample size of patients in this subgroup $(n=89)$, demonstrated that, compared to non-diabetic patients with prior CABG, subsequent DES in the NCA and grafts of diabetic patients resulted in worse outcomes, consistent with Ahmed and Ashfaq's studies. According to the 2018 ESC/EACTS Guidelines for myocardial revascularization [2], it is recommended that PCI in the NCA should be considered rather than PCI in an SVG graft, because that is associated with a high risk of periprocedural MI [28] and worse long-term outcomes such as all-cause death, MI or revascularization [29] for no-reflow, subsequent in-stent restenosis, distant target lesions and excessive tortuosity [8], especially for PCI in an SVG of a diabetic patient for graft atherosclerosis with a larger necrotic core and friable plaques [20].

In this study, we also provided follow-up outcomes of patients with IR vs CR by PCI. Achieving CR of all significantly obstructed coronary artery has been an established goal of PCI, and more recent data demonstrate a salutary effect of CR following PCI on long-term outcomes. IR is associated with increased mortality following PCI, as well as with an increased incidence of MI, repeat revascularization, and MACCEs [30]. Though, in our study the sample size of patients with IR was too small (5.1\%), which would influence statistical results, we still believed that it made a little sense, compared with patients with $\mathrm{CR}$, patients with IR were more likely to have MACEs (40.5\%), cardiac death (8.1\%) and MI (21.6\%), though there were no significant difference between two groups. Further randomized controlled trial study with a larger sample size and longer follow-up may be required for patient with prior CABG.

\section{Limitations}

Firstly, this was a retrospective observational single-center study and so is subject to all the limitations of observational single-center studies, such as patient selection and a single therapeutic method, which might affect the results. Secondly, the angiography film results were analyzed by one cardiac surgeon and one cardiologist. Thirdly, the classification of graft lesions was in reference to the evaluation criteria of native vessels. Fourthly, the decision to perform PCI for each patient was taken by 2 operators, mostly based on an evaluation of the CAG results. Fifthly, 6 non-DM patients who had diabetes during the followup period were excluded from this study. Sixthly, we didn't do PS matching for sub-group analysis. Despite these limitations, the results were derived from the largest angiographic study in patients with prior CABG so far published. In addition, the statistical analyses utilized rigorous methodology.

\section{Conclusions}

Compared to non-diabetic patients with prior CABG, subsequent PCI in an NCA with DES in diabetic patients appears to result in a similar incidence of overall MACEs, cardiac death, MI, HF and repeated revascularization, suggesting that DES may be considered the default option for these patient populations. We also found that hypertension, prior $\mathrm{HF}, \mathrm{LVEF}<50 \%$ and aspiration of thrombus are predictive for overall rate of MACE in diabetic patients with prior CABG. Patients that were administered statins were less likely to experience MACEs.

\section{Supplementary information}

Supplementary information accompanies this paper at https://doi.org/10 1186/s12872-020-01447-8.

\begin{abstract}
Additional file 1: Table S1. Comparison of baseline characteristics of two subgroups of patients with $\mathrm{PCl}$ in different target vessels. Table S2. Procedural baseline characteristics of two subgroups of patients with $\mathrm{PCl}$ in different target vessels. Table S3. Procedure-related complications of two subgroups of patients with $\mathrm{PCl}$ in different target vessels. Table S4. Follow-up outcomes of subgroup of patients with PCl in NCA only. Table S5. Follow-up outcomes of subgroup of patients with PCI in NCA and Graft. Table S6. Comparison of baseline characteristics of two subgroups of patients with $\mathrm{PCI}$ with different generation DESs. Table S7. Procedural baseline characteristics of two subgroups of patients with $\mathrm{PCl}$ with different generation DESs. Table S8. Procedure-related complications of two subgroups of patients with PCI with different generation DESs. Table S9. Follow-up outcomes of patients with first-generation DESS PCI. Table

S10. Follow-up outcomes of patients with second-generation DESs PCI.

Table S11. Follow-up outcomes of patients with incomplete revascularization vs complete revascularization.
\end{abstract}

Additional file 2: Figure S1. Incidence of MACEs (A), cardiac death (B), $\mathrm{MI}(\mathrm{C}), \mathrm{HF}(\mathrm{D})$ or revascularization (E) of patients with $\mathrm{PCl}$ in only NCA (No DM vs DM) using the Kaplan-Meier method. $P$ value was calculated by log-rank test. DM = diabetes mellitus, HF = acute heart failure, NCA = native coronary artery, MACEs = major adverse cardiac events, $\mathrm{MI}=$ myocardial infarction.

Additional file 3: Figure S2. Incidence of MACEs (A), cardiac death (B), $\mathrm{MI}(\mathrm{C}), \mathrm{HF}(\mathrm{D})$ or revascularization (E) of patients with $\mathrm{PCl}$ in NCA and graft (No DM vs DM) using the Kaplan-Meier method. $P$ value was calculated by log-rank test. $\mathrm{DM}=$ diabetes mellitus, $\mathrm{HF}=$ acute heart failure, NCA = native coronary artery, MACEs = major adverse cardiac events, $\mathrm{MI}=$ myocardial infarction.

Additional file 4: Figure S3. Incidence of MACEs (A), cardiac death (B), $\mathrm{MI}(\mathrm{C}), \mathrm{HF}(\mathrm{D})$ or revascularization (E) of patients with first-generation DES PCI (No DM vs DM) using the Kaplan-Meier method. $P$ value was calculated by log-rank test. DM = diabetes mellitus, $\mathrm{HF}=$ acute heart failure, $\mathrm{NCA}=$ native coronary artery, MACEs = major adverse cardiac events, $\mathrm{MI}=$ myocardial infarction.

Additional file 5: Figure S4. Incidence of MACEs (A), cardiac death (B), $\mathrm{MI}(\mathrm{C}), \mathrm{HF}(\mathrm{D})$ or revascularization (E) of patients with second-generation DES PCI (No DM vs DM) using the Kaplan-Meier method. $P$ value was calculated by log-rank test. DM = diabetes mellitus, HF = acute heart failure, $\mathrm{NCA}=$ native coronary artery, MACEs = major adverse cardiac events, $\mathrm{MI}=$ myocardial infarction.

\section{Abbreviations}

AHF: Acute heart failure; AMI: Acute myocardial infarction; BMI: Body mass index; CABG: Coronary artery bypass graft; CTO: Chronic total occlusion; CVA: Cerebrovascular accident; DES: Drug-eluting stent; DM: Diabetes 
mellitus; HbA1c: Glycosylated hemoglobin; HF: Heart failure; LVEF: Left ventricular ejection fraction; NCA: Native coronary artery; MACEs: Major adverse cardiac events; PCI: Percutaneous coronary intervention; PVD: Peripheral vascular disease; SA: Stable angina; SVG: Saphenous vein graft; UA: Unstable angina

\section{Acknowledgements}

Not applicable.

\section{Authors' contributions}

The most important acknowledgment is to the participants in the study at Anzhen Hospital. JG W obtained funding. JG W, XL M, and R D designed the study. PF C, YC Z, CW Z, M X and Q Y collected and analyzed the data. XL M drafted the manuscript. The authors read and approved the manuscript.

\section{Funding}

This study was supported by grants from the National Natural Science Foundation of China $(81770320,81370294,81570291)$. Professor Jiangang Wang owned the funding, a member of designers of this study.

\section{Availability of data and materials}

The datasets generated and analyzed for this current study are available from the corresponding author upon reasonable request.

\section{Ethics approval and consent to participate}

Not applicable.

\section{Consent for publication}

Not applicable.

\section{Competing interests}

The authors declare that they have no competing interests.

\section{Author details}

${ }^{1}$ Department of Cardiac Surgery, Beijing Anzhen Hospital, Capital Medical University, Beijing, China. ${ }^{2}$ Center for Cardiac Intensive Care, Beijing Anzhen Hospital, Capital Medical University, Beijing, China.

Received: 22 August 2019 Accepted: 25 March 2020

Published online: 06 April 2020

\section{References}

1. O'Donoghue ML, Vaidya A, Afsal R, et al. An invasive or conservative strategy in patients with diabetes mellitus and non-ST-segment elevation acute coronary syndromes: a collaborative meta-analysis of randomized trials. J Am Coll Cardiol. 2012;60:106-11.

2. Neumann F-J, Sousa-Uva M, Ahlsson A, et al. 2018 ESC/EACTS quidelines on myocardial revascularization: the task force on myocardial revascularization of the European Society of Cardiology (ESC) and the European Association for Cardio-Thoracic Surgery (EACTS) developed with the special contribution of the European Association of Percutaneous Cardiovascular Interventions (EAPCI). Eur Heart J. 2018:00:1-96.

3. Qi X, Xu M, Yang H, Zhou L, et al. Comparing mortality and myocardial infarction between coronary artery bypass grafting and drug-eluting stenting in patients with diabetes mellitus and multivessel coronary artery disease: a meta-analysis. Arch Med Sci. 2014;10(3):411-8.

4. Kappetein AP, Head SJ, Morice MC, et al. Treatment of complex coronary artery disease in patients with diabetes: 5 -year results comparing outcomes of bypass surgery and percutaneous coronary intervention in the SYNTAX trial. Eur J Cardiothorac Surg. 2013;43(5):1006-13.

5. Hlatky MA, Boothroyd DB, Bravata DM, et al. Coronary artery bypass surgery compared with percutaneous coronary interventions for multivessel disease: a collaborative analysis of individual patient data from ten randomised trials. Lancet. 2009:373:1190-7.

6. Shah $R$, Yang $Y$, Bentley JP, et al. Comparative effectiveness of coronary artery bypass grafting (CABG) surgery and percutaneous coronary intervention $(\mathrm{PCI})$ in elderly patients with diabetes. Curr Med Res Opin. 2016;32(11):1891-8.

7. Brener SJ, Lytle BW, Casserly IP, et al. Predictors of revascularization method and long-term outcome of percutaneous coronary intervention or repeat coronary bypass surgery in patients with multivessel coronary disease and previous coronary bypass surgery. Eur Heart J. 2006:27:413-8.

8. Scarsini R, Zivelonghi $C$, Pesarini $G$, et al. Repeat revascularization: percutaneous coronary intervention after coronary artery bypass graft surgery. Cardiovasc Revasc Med. 2016;17(4):272-8.

9. Boyden TF, Nallamothu BK, Moscucci $M$, et al. Meta-analysis of randomized trials of drug-eluting stents versus bare metal stents in patients with diabetes mellitus. Am J Cardiol. 2007;99:1399-402.

10. Mulukutla SR, Vlachos HA, Marroquin OC, et al. Impact of drug-eluting stents among insulin-treated diabetic patients: a report from the National Heart, Lung, and Blood Institute dynamic registry. JACC Cardiovasc Interv. 2008;1:139-47.

11. Stenestrand U, James SK, Lindbäck J, et al. Safety and efficacy of drugeluting vs. bare metal stents in patients with diabetes mellitus: long-term follow-up in the Swedish coronary angiography and angioplasty registry (SCAAR). Eur Heart J. 2010:31:177-86.

12. Pendyala LK, Loh JP, Kitabata H, Minha S, et al. The impact of diabetes mellitus on long-term clinical outcomes after percutaneous coronary saphenous vein graft interventions in the drug-eluting stent era. J Interv Cardiol. 2014:27(4):391-8

13. Nakamura Y, Asaumi Y, Miyagi T, et al. Comparison of long-term mortality in patients with previous coronary artery bypass grafting undergoing percutaneous coronary intervention with versus without optimal medical therapy. Am J Cardiol. 2018;122:206-12.

14. Honda S, Asaumi Y, Yamane T, et al. Trends in the clinical and pathological characteristics of cardiac rupture in patients with acute myocardial infarction over 35 years. J Am Heart Assoc. 2014;3:e000984.

15. Moreno PR, Murcia AM, Palacios IF, et al. Coronary composition and macrophage infiltration in atherectomy specimens from patients with diabetes mellitus. Circulation. 2000;102:2180-4.

16. Marso SP, Mercado N, Maehara A, et al. Plaque composition and clinical outcomes in acute coronary syndrome patients with metabolic syndrome or diabetes. JACC Cardiovasc Imaging. 2012;5:S42-52.

17. Schofield I, Malik R, Izzard A, et al. Vascular structural and functional changes in type 2 diabetes mellitus: evidence for the roles of abnormal myogenic responsiveness and dyslipidemia. Circulation. 2002;106:3037-43.

18. Haffner SM, Lehto S, Rönnemaa T, et al. Mortality from coronary heart disease in subjects with type 2 diabetes and in nondiabetic subjects with and without prior myocardial infarction. N Engl J Med. 1998:339:229-34.

19. Guandalini GS, Bangalore S. The potential effects of new stent platforms for coronary revascularization in patients with diabetes. Can J Cardiol. 2018; 34(5):653-64.

20. Baars $T$, Konorza $T$, Kahlert $P$, et al. Coronary aspirate TNF a reflects saphenous vein bypass graft restenosis risk in diabetic patients. Cardiovasc Diabetol. 2013:12:12

21. Detre KM, Lombardero MS, Brooks MM, et al. The effect of previous coronary-artery bypass surgery on the prognosis of patients with diabetes who have acute myocardial infarction. Bypass angioplasty revascularization investigation investigators. N Engl J Med. 2000;342(14):989-97.

22. The BARI Investigators. Influence of diabetes on 5-year mortality and morbidity in a randomized trial comparing CABG and PTCA in patients with multivessel disease: the bypass angioplasty revascularization investigation (BARI). Circulation. 1997;96:1761-9.

23. Tailakh MA, Friger M, Zahger $D$, et al. Prospective study of the impact of diabetes mellitus newly diagnosed by glycated hemoglobin on outcomes in patients undergoing percutaneous coronary intervention. Eur J Intern Med. 2017:37:69-74.

24. De Luca G, Dirksen MT, Spaulding C, et al. Impact of diabetes on long-term outcome after primary angioplasty: insights from the DESERT cooperation. Diabetes Care. 2013;36(4):1020-5.

25. Wang $\mathrm{H}$, Gao Z, Song $Y$, et al. Impact of diabetes mellitus on percutaneous coronary intervention in Chinese patients: a large single-center data. Angiology. 2018;69(6):540-7.

26. Ahmed JM, Hong MK, Mehran R, et al. Influence of diabetes mellitus on early and late clinical outcomes in saphenous vein graft stenting. J Am Coll Cardiol. 2000:36(4):1186-93.

27. Ashfaq S, Ghazzal Z, Douglas JS, et al. Impact of diabetes on five-year outcomes after vein graft interventions performed prior to the drug-eluting stent era. J Invasive Cardiol. 2006;18(3):100-5.

28. Coolong A, Baim DS, Kuntz RE, et al. Saphenous vein graft stenting and major adverse cardiac events: a predictive model derived from a pooled analysis of 3958 patients. Circulation. 2008:117:790-7. 
29. Brilakis ES, O'Donnell Cl, Penny W, et al. Percutaneous coronary intervention in native coronary arteries versus bypass grafts in patients with prior coronary artery bypass graft surgery: insights from the veterans affairs clinical assessment, reporting, and tracking program. JACC Cardiovasc Interv. 2016;9(9):884-93.

30. Kereiakes DJ. Reassessing the importance of complete versus incomplete coronary revascularization. Rev Cardiovasc Med. 2014;15(1):24-30.

\section{Publisher's Note}

Springer Nature remains neutral with regard to jurisdictional claims in published maps and institutional affiliations.

Ready to submit your research? Choose BMC and benefit from:

- fast, convenient online submission

- thorough peer review by experienced researchers in your field

- rapid publication on acceptance

- support for research data, including large and complex data types

- gold Open Access which fosters wider collaboration and increased citations

- maximum visibility for your research: over $100 \mathrm{M}$ website views per year

At $\mathrm{BMC}$, research is always in progress.

Learn more biomedcentral.com/submissions 